Research Article

\title{
Entropy Analysis on a Three-Dimensional Wavy Flow of Eyring-Powell Nanofluid: A Comparative Study
}

\author{
Arshad Riaz, ${ }^{1}$ Ahmed Zeeshan, ${ }^{2}$ and M. M. Bhatti ${ }^{3}{ }^{3}$ \\ ${ }^{1}$ Department of Mathematics, Division of Science and Technology, University of Education, Lahore 54770, Pakistan \\ ${ }^{2}$ Department of Mathematics and Statistics, Faculty of Basic and Applied Sciences, International Islamic University, \\ Islamabad 44000, Pakistan \\ ${ }^{3}$ College of Mathematics and Systems Science, Shandong University of Science and Technology, Qingdao, \\ Shandong 266590, China
}

Correspondence should be addressed to M. M. Bhatti; muhammad09@shu.edu.cn

Received 3 October 2020; Revised 7 November 2020; Accepted 24 February 2021; Published 5 March 2021

Academic Editor: Georgios I. Giannopoulos

Copyright (c) 2021 Arshad Riaz et al. This is an open access article distributed under the Creative Commons Attribution License, which permits unrestricted use, distribution, and reproduction in any medium, provided the original work is properly cited.

The thermal management of a system needs an accurate and efficient measurement of exergy. For optimal performance, entropy should be minimized. This study explores the enhancement of the thermal exchange and entropy in the stream of Eyring-Powell fluid comprising nanoparticles saturating the vertical oriented dual cylindrical domain with uniform thermal conductivity and viscous dissipation effects. A symmetrical sine wave over the walls is used to induce the flow. The mathematical treatment for the conservation laws are described by a set of PDEs, which are, later on, converted to ordinary differential equations by homotopy deformations and then evaluated on the Mathematica software tool. The expression of the pressure rise term has been handled numerically by using numerical integration by Mathematica through the algorithm of the Newton-Cotes formula. The impact of the various factors on velocity, heat, entropy profile, and the Bejan number are elaborated pictorially and tabularly. The entropy generation is enhanced with the variation of viscous dissipation but reduced in the case of the concentration parameter, but viscous dissipation reveals opposite findings for the Newtonian fluid. From the abovementioned detailed discussion, it can be concluded that Eyring-Powell shows the difference in behavior in the entropy generation and in the presence of nanoparticles due to the significant dissipation effects, and also, it travels faster than the viscous fluid. A comparison between the Eyring-Powell and Newtonian fluid are also made for each pertinent parameter through special cases. This study may be applicable for cancer therapy in biomedicine by nanofluid characteristics in various drugs considered as a non-Newtonian fluid.

\section{Introduction}

Nonlinear fluid models are the center of consideration of several theoretical and experimental evaluations due to their massive applicability in the engineering sciences, biomechanics, and mechanical manufacturing such as the petroleum industry, flow of blood in the body, and transport of sewage. Working in the domain of nonlinear fluid flows creates challenges to the mathematician and simulation engineers with its diversity and complexity. As the complexity of such fluids offers no unique constitutive equation which encounters all the properties, for such fluids, several non-Newtonian models are presented in
[1-4]. The polymeric liquids reveal diverse rheological attributes apparent in the flow, such as polymer accumulation, mechanical mortification, and solvent composition. [5]. Kwack [6] explained that all these polymer properties influence the elasticity of the fluids. Mathematical models such as the Carreau fluid model [7], Ellis fluid model [8], and Powell-Eyring fluid model [9] effectively discuss elastic properties of the fluid. Out of these models, the Powell-Eyring model stands out because of its fluid elasticity property and ease for its applicability to both experimental and theoretical studies. These advantages lead many scientists to unveil these hidden properties of such fluids in different flow situations. Patel and 
Timol [10] found a numerical solution to debate on the characteristics of the Powell-Eyring type model. They used the so-called MSABC scheme to find an asymptotically convergent solution. Hayat et al. [11] found a series solution using the so-called HAM to define the momentum and heat transfer about the stagnation point for the Powell-Eyring fluid. They also analyzed melting heat transfer effects. Jalil et al. [12] discovered self-similar solutions of parallel free stream flow of this fluid. The flow is considered on a moving plate. Islam et al. [13] considered the flow of the Powell-Eyring fluid in slider bearings. They employed HPM to develop the analytic series solution of the current flow. Hina et al. [14] elaborated the study of Powell-Eyring fluids between curved compliant walls of the channel. The effects on temperature variations are also discussed. Hayat et al. [15] evaluated MHD Powell-Eyring liquid flow on a radially stretching cylinder. They exclusively focused on the effects of Newtonian heating.

In recent times, the study of flows driven by peristaltic waves has become more and more popular in physiology and biomedical industry. The idea of peristalsis was driven from the food transport duct in the human body. Other than food transport from the mouth to stomach, this phenomenon can be seen in the spermatozoa movement in the male genital region and ovum in the females and also in urine travelling and chyme movement through the gastrointestinal tract, etc. In industry, machine-like rollers and finger pumps and blood filtration devices in dialysis employ the mechanism of peristalsis. Shapiro [16] and Latham [17] presented the idea of peristalsis theoretically and verified experimentally. Ever since, numerous studies, both theoretically and experimentally, have been published in the domain of nonlinear fluids [18-20]. The peristaltic mechanism of certain elastic fluids is a matter of great interest. Recent studies are indicative of the importance of these in industrial applications. Ellahi et al. [21] used 3D analysis of a peristaltic stream of Carreau fluid in the magnetic environment. Hayat et al. [22] worked with the Carreau-Yasuda fluid with nanosized particle theoretically. They considered that flow is driven due to the sinusoidal motion of the wall. Also, they examined both convective and no-mass flux on the wall. Prakash and Tripathi [23] examined the effects of the electric double layer on Williamson ionic liquid with homogeneously distributed particles of nanosize. They considered a tapered channel with peristaltic waves on the wall. Effects of thermal radiation are also discovered. Zeeshan et al. [24] focused on the flow of the bio-Jeffrey fluid and the sustainability character in the human body. Tanveer et al. [25] checked the flow through a curved channel. They revealed mixed convection impacts and elastic properties of the walls on pumping transport of Eyring-Powell liquid with nanomaterial. Bhatti et al. [26] presented the effects of the Darcy porous medium of a two-phase nanofluid in a channel.

The applications such as paper drying, thermal coating, hot rolling, and glass fiber stretching divert one's focus towards the heat transfer of non-Newtonian fluids. Motivated by this, it stimulates an extensive literature in this field [27-30] which includes analysis in flexible channels. Presence of nanoparticles shows a positive trend in enhancing the heat transfer of fluid. The theme of nanofluids is the concept of Choi [31]. The concept was to add nanosized particles in a fluid which has low thermal conductivity. Hence, fluid behavior and metallic conductivity both combine to obtain an enhanced heat conductor which can be transported like a fluid. It was one of the promising aspects of such fluids. Many mathematical models using continuum formulation [32] or twophase suspension models [33] are developed over the years now. One such model due to Buongiorno [34] was developed in many ways. Some researchers [35-39] revealed the basics of nanofluids' contribution in different geometries with the peristaltic wave.

It is essential to generate the systems or the machines which perform and help in efficient energy transport. Such a machine is broadly utilized in power plants, manufacturing plants, and transportation. The warm productivity of the framework relies upon the material used for thermal vitality. It is fundamental to understand the factor which diminishes warm proficiency. As indicated by the laws of thermodynamics, vitality of the framework stays to save, yet can be changed over into different structures for the utility. All the more regularly, we state all the energies of the framework are spent in accomplishing work or to expand the temperature of the body. Numerous frictional powers emerging from the attractive field, permeable spaces, and so on are explained in the improvement of the temperature of the framework. The warm messiness in the framework is consistently on the ascent. Realizing the elements engaged with entropy rise is consistently imperative to get ideal warm effectiveness of the framework. Ascend being used of nanofluids is one approach to decrease the loss of vitality. Entropy generation and exergy are one of the most dynamic examination fields in thermodynamics. Most recently, $\mathrm{Naz}$ et al. [40] optimized entropy generated in the flow of pseudoplastic fluid. The fluid also comprises of microorganisms. Abbas et al. [41] analyzed the entropy generated theoretically in the stream of magnetized nanofluid. They use statistical techniques to get results. Abbas et al. [42] also analyzed entropy for peristaltic flow of nanofluids. Ellahi et al. [43] extended the work with a porous medium. Hayat et al. [44] use modified Darcy's porous medium and the effects of the endoscope.

The limited literature on entropy generation of nanoelastic fluid, useful characteristics of the EyringPowell model, and the applicability in the industrial usage of such flows is the main motivation for the current analysis. With the cited literature in mind, the existing study designed to investigate the distribution of nanoparticles in a Powell-Eyring viscoelastic fluid having enhanced elastic properties and viscous dissipation effects through a cylindrical structure with the peristaltic wave is focused. Energy loss in the transport is also an area of interest. The observations for the Newtonian fluid are also taken into consideration as a limiting case of the readings. The obtained PDEs are simplified with the help of laminar 
movement of the liquid and small wave amplitude approximations and then solved analytically. The results are elaborated comprehensively and displayed in the graphical way. Conclusions which highlight the key findings are placed in the end.

\section{Mathematical Treatment}

We take the peristaltic flow of an Eyring-Powell liquid situated in an annular part of two eccentric cylinders by introducing nanoparticles. We also consider the term of viscous dissipation and entropy generation occurring during the process due to the irreversibilities of viscous factors, thermal analysis, and mass exchanging phenomenon of nanoparticles. The geometry is assumed to be having flexible outer surfaces. The flow is taken inside the annular region of two eccentric annuli with eccentricity $\epsilon$. Moreover, the inner cylinder is considered as rigid with radius $\delta$, and the outer cylinder with radius $a$ is producing a sinusoidal wave propagating along the axial root. The walls of the inner and outer annuli have been described in [30]. The conservation laws for an incompressible and unsteady flow of non-Newtonian nanofluid are described in $[30,35]$. The temperatures and nanoparticle concentrations at the inner cylinder are fixed at the amount of $T_{o}$ and $C_{o}$, while those of the outer cylinder are maintained at $T_{1}$ and $C_{1} \simeq$ accordingly. The mathematical model of the stress matrix representing the Eyring-Powell fluid is described as follows [15]:

$$
S_{\mathrm{LM}}=\mu \frac{\partial V_{p}}{\partial x^{i}}+\frac{1}{\beta} \operatorname{Arcsinh}\left(c^{-1} \frac{\partial V_{p}}{\partial x^{i}}\right),
$$

$\operatorname{Arcsinh}\left(c^{-1} \frac{\partial V_{p}}{\partial x^{i}}\right) \approx c^{-1} \frac{\partial V_{p}}{\partial x^{i}}-\frac{1}{6}\left(c^{-1} \frac{\partial V_{p}}{\partial x^{i}}\right)^{3}+O\left|c^{-1} \frac{\partial V_{p}}{\partial x^{i}}\right|^{5}$

where $\mu$ represents the shear viscosity coefficient and $\beta$ and $c$ are the material constants. Now, we introduce local wave frame coordinates $(r, \theta, z)$ running with the same wave velocity relative to the fixed frame $\left(x^{1}, x^{2}, x^{3}\right)$ through the following transformations:

$$
\begin{aligned}
z & =x^{3}-c_{1} t, \\
r & =x^{1}, \\
\theta & =x^{2}, \\
w & =\underline{W}-c_{1}, \\
p & =P, \\
u & =\underline{U}, \\
S_{\mathrm{LM}} & =s_{\operatorname{lm}} .
\end{aligned}
$$

We use the following dimensionless parameters in the problem:

$$
\begin{aligned}
& p^{\prime}=\frac{a^{2}}{\mu c_{1} \lambda} p, \\
& w^{\prime}=\frac{w}{c_{1}}, \\
& u^{\prime}=\frac{\lambda}{a c_{1}} u \text {, } \\
& V^{\prime}=\frac{V}{c_{1}}, \\
& z^{\prime}=\frac{z}{\lambda}, \\
& r^{\prime}=\frac{r}{a}, \\
& \theta^{\prime}=\theta \text {, } \\
& t^{\prime}=\frac{c}{\lambda} t, \\
& \phi=\frac{b}{a}, \\
& \epsilon^{\prime}=\frac{\epsilon}{a}, \\
& R_{e}=\frac{\rho c_{1} a}{\mu}, \\
& \delta^{\prime}=\frac{\delta}{a}, \\
& \bar{\theta}=\frac{T-T_{o}}{T_{1}-T_{o}}, \\
& \delta_{o}=\frac{a}{\lambda} \text {, } \\
& r_{1}^{\prime}=r_{1} \text {, } \\
& r_{2}^{\prime}=\frac{r_{2}}{a} \text {, } \\
& \sigma=\frac{C-C_{o}}{C_{1}-C_{o}}, \\
& P_{r}=\frac{\mu}{\rho \alpha} \text {, } \\
& S_{c}=\frac{\mu}{\rho D_{B}}, \\
& \alpha_{f}=\frac{k}{(\rho c)_{f}} \text {, } \\
& \tau=\frac{(\rho c)_{p}}{(\rho c)_{f}}, \\
& B_{r}=\frac{\rho_{f} g \alpha a^{2}}{\mu c}\left(T_{1}-T_{o}\right), \\
& G_{r}=\frac{\rho_{f} g \alpha a^{2}}{\mu c}\left(C_{1}-C_{o}\right), \\
& N_{b}=\frac{\tau D_{B}}{\alpha_{f}}\left(C_{1}-C_{o}\right),
\end{aligned}
$$




$$
\begin{aligned}
N_{t} & =\frac{\tau D_{T}}{T_{o} \alpha_{f}}\left(T_{1}-T_{o}\right), \\
\grave{S} & =\frac{a}{\mu c_{1}} S, \\
G_{c} & =\frac{\mu c^{2}}{k\left(T_{1}-T_{o}\right)}, \\
M & =\frac{1}{\beta \mu c} \\
K & =\frac{M c_{1}^{2}}{6 c^{2} a^{2}},
\end{aligned}
$$

where $V, \phi, R_{e}, \delta_{o}, P_{r}, N_{b}, N_{t}, G_{c}, G_{r}$, and $B_{r}$ evaluate the velocity of the inner rod, amplitude fraction, Reynolds number factor, nondimensional wave number, the Prandtl number, the Brownian motion parameter, the thermophoresis constant parameter, Brinkman number, localized temperature Grashof number, and localized nanoparticles Grashof number, separately. After employing the abovementioned nondimensional parameters and wave frame, we approach to the following dimensionless form of the equations of motion defined in [30] under the physiological limitations of long wavelength $(\lambda \longrightarrow \infty)$ and low Reynolds number $\left(R_{e} \longrightarrow 0\right)$ :

$$
\frac{\partial u^{\prime}}{\partial r^{\prime}}+\frac{\partial w^{\prime}}{\partial z^{\prime}}+\frac{u^{\prime}}{r^{\prime}}=0
$$

$$
\begin{aligned}
0= & -\frac{\partial p^{\prime}}{\partial z^{\prime}}+\frac{1}{r^{\prime}} \frac{\partial\left(r^{\prime} S_{r z}^{\prime}\right)}{\partial r^{\prime}}+\frac{1}{r^{\prime}} \frac{\partial S_{\theta z}^{\prime}}{\partial \theta^{\prime}}+G r \bar{\theta}+B r \sigma \\
0= & \left(\frac{\partial^{2} \overline{\theta^{\prime}}}{\partial r^{\prime 2}}+\frac{1}{r^{\prime 2}} \frac{\partial^{2} \overline{\theta^{\prime}}}{\partial \theta^{\prime 2}}+\frac{1}{r^{\prime}} \frac{\partial \overline{\theta^{\prime}}}{\partial r^{\prime}}\right)+N_{b}\left(\frac{\partial \sigma}{\partial r^{\prime}} \frac{\partial \overline{\theta^{\prime}}}{\partial r^{\prime}}+\frac{1}{r^{\prime 2}} \frac{\partial \sigma}{\partial \theta^{\prime}} \frac{\partial \overline{\theta^{\prime}}}{\partial \theta^{\prime}}\right)+N_{t}\left(\left(\frac{\partial \overline{\theta^{\prime}}}{\partial r^{\prime}}\right)^{2}+\frac{1}{r^{\prime 2}}\left(\frac{\partial \overline{\theta^{\prime}}}{\partial \theta^{\prime}}\right)\right) \\
& +G_{c}\left(S_{r z}^{\prime} \frac{\partial w^{\prime}}{\partial r^{\prime}}+\frac{1}{r^{\prime}} S_{z \theta}^{\prime} \frac{\partial w^{\prime}}{\partial \theta^{\prime}}\right), \\
0= & \left(\frac{\partial^{2} \sigma}{\partial r^{\prime 2}}+\frac{1}{r^{\prime 2}} \frac{\partial^{2} \sigma}{\partial \theta^{2}}+\frac{1}{r^{\prime}} \frac{\partial \sigma}{\partial r^{\prime}}\right)+\frac{N_{t}}{N_{b}}\left(\frac{\partial^{2} \overline{\theta^{\prime}}}{\partial r^{\prime 2}}+\frac{1}{r^{\prime 2}} \frac{\partial^{2} \overline{\theta^{\prime}}}{\partial \theta^{\prime 2}}+\frac{1}{r^{\prime}} \frac{\partial \overline{\theta^{\prime}}}{\partial r^{\prime}}\right) .
\end{aligned}
$$

The needed nondimensional components of stress tensor $S_{\mathrm{LM}}$ calculated from relation (1) can be collected as follows:

$$
\begin{aligned}
& S_{r z}^{\prime}=\frac{\partial w^{\prime}}{\partial r^{\prime}}+M \frac{\partial w^{\prime}}{\partial r^{\prime}}-K\left(\frac{\partial w^{\prime}}{\partial r^{\prime}}\right)^{3}, \\
& S_{z \theta}^{\prime}=\frac{1}{r^{\prime}} \frac{\partial w^{\prime}}{\partial \theta^{\prime}}+\frac{M}{r^{\prime}} \frac{\partial w^{\prime}}{\partial \theta^{\prime}}-K\left(\frac{1}{r^{\prime}} \frac{\partial w^{\prime}}{\partial \theta^{\prime}}\right)^{3}, \\
& S_{z \theta}^{\prime}=0 .
\end{aligned}
$$

Now, we use $S_{r z}^{\prime}, S_{\theta z}^{\prime}$, and $S_{z \theta}^{\prime}$ in (6) and (7) and also omit the prime symbols to have the resulting structure as follows:

$$
\begin{aligned}
\frac{1}{(1+M)} p^{\prime}(z)= & \frac{\partial^{2} w}{\partial r^{2}}+\frac{1}{r} \frac{\partial w}{\partial r}+\frac{1}{r^{2}} \frac{\partial^{2} w}{\partial \theta^{2}}-\frac{3 K}{(1+M)}\left(\frac{\partial w}{\partial r}\right)^{2} \frac{\partial^{2} w}{\partial r^{2}}-\frac{K}{r(1+M)}\left(\frac{\partial w}{\partial r}\right)^{3} \\
& -\frac{3 K}{r^{4}(1+M)}\left(\frac{\partial w}{\partial \theta}\right)^{2} \frac{\partial^{2} w}{\partial \theta^{2}}+\frac{1}{(1+M)}(G r \bar{\theta}+B r \sigma), \\
0= & \left(\frac{\partial^{2} \bar{\theta}}{\partial r^{2}}+\frac{1}{r} \frac{\partial \bar{\theta}}{\partial r}+\frac{1}{r^{2}} \frac{\partial^{2} \bar{\theta}}{\partial \theta^{2}}\right)+N_{b}\left(\frac{\partial \sigma}{\partial r} \frac{\partial \bar{\theta}}{\partial r}+\frac{1}{r^{2}} \frac{\partial \sigma}{\partial \theta} \frac{\partial \bar{\theta}}{\partial \theta}\right)+N_{t}\left(\left(\frac{\partial \bar{\theta}}{\partial r}\right)^{2}+\frac{1}{r^{2}}\left(\frac{\partial \bar{\theta}}{\partial \theta}\right)^{2}\right) \\
& +G_{c}\left(\left(\frac{\partial w}{\partial r}\right)^{2}+M\left(\frac{\partial w}{\partial r}\right)^{2}-K\left(\frac{\partial w}{\partial r}\right)^{4}\right),
\end{aligned}
$$




$$
0=\left(\frac{\partial^{2} \sigma}{\partial r^{2}}+\frac{1}{r} \frac{\partial \sigma}{\partial r}+\frac{1}{r^{2}} \frac{\partial^{2} \sigma}{\partial \theta^{2}}\right)+\frac{N_{t}}{N_{b}}\left(\frac{\partial^{2} \bar{\theta}}{\partial r^{2}}+\frac{1}{r} \frac{\partial \bar{\theta}}{\partial r}+\frac{1}{r^{2}} \frac{\partial^{2} \bar{\theta}}{\partial \theta^{2}}\right)
$$

The relevant no-slip walls' conditions in a nondimensional format are described as follows [30]:

$$
\begin{aligned}
w & =\left\{V \text { when } r=r_{1}=\delta+\varepsilon \cos \theta, 0 \text { when } r=r_{2}=1+\phi \cos 2 \pi z\right\}, \\
(\bar{\theta}, \sigma) & =\left\{0 \text { when } r=r_{1}=\delta+\varepsilon \cos \theta, 1 \text { when } r=r_{2}=1+\phi \cos 2 \pi z\right\} .
\end{aligned}
$$

\section{Solution Procedure}

The solutions of the overhead nonlinear partial equations, (10), (11), and (12), are achieved analytically. The deformation expressions for the considering problems are evaluated as follows [30, 37]:

$$
\begin{gathered}
\left(1-q^{\prime \prime}\right)\left(\mathscr{L}[\widetilde{w}]-\mathscr{L}\left[\widetilde{w}_{o}\right]\right)+q^{\prime \prime}\left[\begin{array}{c}
\left.\mathscr{L}[\widetilde{w}]+\frac{1}{r^{2}} \frac{\partial^{2} \widetilde{w}}{\partial \theta^{2}}-\frac{3 K}{(1+M)}\left(\frac{\partial \widetilde{w}}{\partial r}\right)^{2} \frac{\partial^{2} \widetilde{w}}{\partial r^{2}}-\frac{K}{r(1+M)}\left(\frac{\partial \widetilde{w}}{\partial r}\right)^{3}-\frac{3 K}{r^{4}(1+M)}\left(\frac{\partial \widetilde{w}}{\partial \theta}\right)^{2} \frac{\partial^{2} \widetilde{w}}{\partial \theta^{2}}\right)^{\prime}(1+M) \\
\left.+G_{r} \Theta+B r \Omega-p^{\prime}(z)\right)
\end{array}\right] \\
(1-q)\left(\mathscr{L}[\Theta]-\mathscr{L}\left[\tilde{\theta}_{o}\right]\right)+q^{\prime \prime}\left[\begin{array}{c}
\mathscr{L}[\Theta]+\frac{1}{r^{2}} \frac{\partial^{2} \Theta}{\partial \theta^{2}}+N b\left(\frac{\partial \Theta}{\partial r} \frac{\partial \Omega}{\partial r}+\frac{1}{r^{2}} \frac{\partial \Theta}{\partial \theta} \frac{\partial \Omega}{\partial \theta}\right) \\
+N_{t}\left(\left(\frac{\partial \Theta}{\partial r}\right)^{2}+\frac{1}{r^{2}}\left(\frac{\partial \Theta}{\partial \theta}\right)^{2}\right)+G_{c}\left(\left(\frac{\partial \widetilde{w}}{\partial r}\right)^{2}+M\left(\frac{\partial \widetilde{w}}{\partial r}\right)^{2}-K\left(\frac{\partial \widetilde{w}}{\partial r}\right)^{4}\right)
\end{array}\right]=0, \\
(1-q) \mathscr{L}\left[\Omega-\widetilde{\sigma}_{o}\right]+q^{\prime \prime}\left[\mathscr{L}[\Omega]+\frac{1}{r^{2}} \frac{\partial^{2} \Omega}{\partial \theta^{2}}+\frac{N_{t}}{N_{b}}\left(\frac{\partial^{2} \Theta}{\partial r^{2}}+\frac{1}{r^{2}} \frac{\partial^{2} \Theta}{\partial \theta^{2}}+\frac{1}{r} \frac{\partial \Theta}{\partial r}\right)\right]=0 .
\end{gathered}
$$

Let us choose the linear operator as $\mathscr{L}=\left(\partial / \partial r^{2}\right)+$ $(1 / r)(\partial / \partial r)$. We observe the following initial choices for $w, \bar{\theta}$, and $\sigma$ :

$$
\begin{aligned}
\widetilde{w}_{o} & =V\left(\log r-\log r_{2}\right)\left(\log r_{1}-\log r_{2}\right)^{-1}, \\
\bar{\theta}_{o} & =\bar{\sigma}_{o}=\left(\log r_{1}-\log r\right)\left(\log r_{1}-\log r_{2}\right)^{-1} .
\end{aligned}
$$

Now, using the perturbation technique on the parameter $q^{\prime \prime}$, we have

$$
\begin{aligned}
& \widetilde{w}\left(r, \theta, z, q^{\prime \prime}\right)=w_{o}+q^{\prime \prime} w_{1}+\ldots, \\
& \widetilde{\Theta}\left(r, \theta, z, q^{\prime \prime}\right)=\bar{\theta}_{o}+q^{\prime \prime} \bar{\theta}_{1}+\ldots, \\
& \widetilde{\Omega}\left(r, \theta, z, q^{\prime \prime}\right)=\sigma_{o}+q^{\prime \prime} \sigma_{1}+\ldots
\end{aligned}
$$

After substituting the abovementioned equations into equations (14)-(16), the solution of velocity $w_{1}$ contains a very large output, so it is not displayed here, and the solutions of $\bar{\theta}_{1}$ and $\sigma_{1}$ are given in Appendix. Now, for $\left(q^{\prime \prime} \longrightarrow 1\right)$, we approach the final solutions. So, from equations (18)-(20), we get

$$
\begin{gathered}
w(r, \theta, z)=w_{o}+w_{1} \\
\bar{\theta}_{o}(r, \theta, z)=\theta_{o}+\theta_{1} \\
\sigma(r, \theta, z)=\sigma_{o}+\sigma_{1}
\end{gathered}
$$

The instant value of volume rate of flow $\overline{Q^{\prime \prime}}$ is taken by

$$
\overline{Q^{\prime \prime}}=2 \pi \int_{r_{1}}^{r_{2}} r w \mathrm{~d} r
$$

The periodic mean volumetric rate of stream $Q$ is found mathematically as follows [30]:

$$
\overline{Q^{\prime \prime}}(z, t)=\frac{Q}{\pi}-\frac{\phi^{2}}{2}+2 \phi \cos [2 \pi(z-t)]+\phi^{2} \cos ^{2}[2 \pi z] .
$$

One can get the measure of pressure rate $p^{\prime}(z)$ by decoding (22) and (23). The dimensionless pressure rise $\_$pis defined as follows [30]:

$$
\Delta p=\int_{0}^{1} p^{\prime}(z) \mathrm{d} z
$$


The integration is performed numerically by using Newton-Cotes formulas through the NIntegrate tool on Mathematica 7.

\section{Entropy Measures}

The expression of entropy generation under the irreversibility of heat exchange, nanoparticles, and viscous effects of the nanofluid is defined as follows [30]:

$$
\begin{aligned}
S_{\text {gen }}^{\prime}= & \frac{K}{T_{o}^{2}}\left(\left(\frac{\partial T}{\partial r}\right)^{2}+\frac{1}{r^{2}}\left(\frac{\partial T}{\partial \theta}\right)^{2}+\left(\frac{\partial T}{\partial z}\right)^{2}\right)+\frac{D_{B}}{C_{o}}\left(\left(\frac{\partial C}{\partial r}\right)^{2}+\frac{1}{r^{2}}\left(\frac{\partial C}{\partial \theta}\right)^{2}+\left(\frac{\partial C}{\partial z}\right)^{2}\right) \\
& +\frac{D_{B}}{T_{o}}\left(\frac{\partial C}{\partial r} \frac{\partial T}{\partial r}+\frac{1}{r^{2}} \frac{\partial C}{\partial \theta} \frac{\partial T}{\partial \theta}+\frac{\partial C}{\partial z} \frac{\partial T}{\partial z}\right)+\frac{1}{T_{o}}\left(S_{r r} \frac{\partial u}{\partial r}+\frac{1}{r} S_{r \theta} \frac{\partial u}{\partial \theta}+S_{r z}\left(\frac{\partial u}{\partial z}+\frac{\partial w}{\partial r}\right)+\frac{1}{r} S_{z \theta} \frac{\partial w}{\partial \theta}+S_{z z} \frac{\partial w}{\partial z}+\frac{u}{r} S_{\theta \theta}\right)
\end{aligned}
$$

The dimensionless factors used in these equations are considered as follows:

$$
\begin{aligned}
N_{s} & =\frac{S_{\text {gen }}^{\prime}}{S_{G}}, \\
S_{G} & =\frac{K\left(T_{1}-T_{o}\right)^{2}}{a^{2} T_{o}^{2}}, \\
\Gamma & =\frac{D_{B} T_{o}\left(C_{1}-C_{o}\right)}{K\left(T_{1}-T_{o}\right)}, \\
\Lambda & =\frac{T_{1}-T_{o}}{T_{o}}, \\
\Omega & =\frac{\left(C_{1}-C_{o}\right)}{C_{o}} .
\end{aligned}
$$

Now, we substitute the abovementioned manipulated factors in equation (25); we have

$$
\begin{aligned}
N_{s}= & \left(\left(\frac{\partial \bar{\theta}}{\partial r^{\prime}}\right)^{2}+\frac{1}{r^{\prime 2}}\left(\frac{\partial \bar{\theta}}{\partial \theta^{\prime}}\right)^{2}+\delta_{O}^{2}\left(\frac{\partial \bar{\theta}}{\partial z^{\prime}}\right)^{2}\right)+\frac{\Gamma \Lambda}{\Omega}\left(\left(\frac{\partial \sigma}{\partial r^{\prime}}\right)^{2}+\frac{1}{r^{\prime 2}}\left(\frac{\partial \sigma}{\partial \theta^{\prime}}\right)^{2}+\delta_{O}^{2}\left(\frac{\partial \sigma}{\partial z^{\prime}}\right)^{2}\right) \\
& +\Gamma\left(\frac{\partial \sigma}{\partial r^{\prime}} \frac{\partial \bar{\theta}}{\partial r^{\prime}}+\frac{1}{r^{\prime 2}} \frac{\partial \sigma}{\partial \theta^{\prime}} \frac{\partial \bar{\theta}}{\partial \theta^{\prime}}+\delta_{O}^{2} \frac{\partial \sigma}{\partial z^{\prime}} \frac{\partial \bar{\theta}}{\partial z^{\prime}}\right) \\
& +\frac{G_{c}}{\Omega}\left(\delta_{o} S_{r r}^{\prime} \frac{\partial u^{\prime}}{\partial r^{\prime}}+\delta_{o} \frac{1}{r^{\prime}} S_{r \theta}^{\prime} \frac{\partial u^{\prime}}{\partial \theta^{\prime}}+S_{r z}^{\prime}\left(\delta_{O}^{2} \frac{\partial u^{\prime}}{\partial z^{\prime}}+\frac{\partial w^{\prime}}{\partial r^{\prime}}\right)+\frac{1}{r^{\prime}} S_{z \theta}^{\prime} \frac{\partial w^{\prime}}{\partial \theta^{\prime}}+\delta_{o} S_{z z}^{\prime} \frac{\partial w^{\prime}}{\partial z^{\prime}}+\delta_{o} \frac{u^{\prime}}{r^{\prime}} S_{\theta \theta}^{\prime}\right) .
\end{aligned}
$$

Incorporating the lubrication strategy in the abovementioned equation, it will take the form

$$
\begin{aligned}
N_{s}= & \left(\left(\frac{\partial \bar{\theta}}{\partial r^{\prime}}\right)^{2}+\frac{1}{r^{\prime 2}}\left(\frac{\partial \bar{\theta}}{\partial \theta^{\prime}}\right)^{2}\right)+\frac{\Gamma \Lambda}{\Omega}\left(\left(\frac{\partial \sigma}{\partial r^{\prime}}\right)^{2}+\frac{1}{r^{\prime 2}}\left(\frac{\partial \sigma}{\partial \theta^{\prime}}\right)^{2}\right) \\
& +\Gamma\left(\frac{\partial \sigma}{\partial r^{\prime}} \frac{\partial \bar{\theta}}{\partial r^{\prime}}+\frac{1}{r^{\prime 2}} \frac{\partial \sigma}{\partial \theta^{\prime}} \frac{\partial \bar{\theta}}{\partial \theta^{\prime}}\right)+\frac{G_{c}}{\Omega}\left(S_{r z}^{\prime} \frac{\partial w^{\prime}}{\partial r^{\prime}}+\frac{1}{r^{\prime}} S_{z \theta}^{\prime} \frac{\partial w^{\prime}}{\partial \theta^{\prime}}\right)
\end{aligned}
$$


Now, using the values of $S_{r z}^{\prime}$ and $S_{z \theta}^{\prime}$ in the abovementioned equation,

$$
\begin{aligned}
N_{s}= & \left(\left(\frac{\partial \bar{\theta}}{\partial r^{\prime}}\right)^{2}+\frac{1}{r^{\prime 2}}\left(\frac{\partial \bar{\theta}}{\partial \theta^{\prime}}\right)^{2}\right)+\frac{\Gamma \Lambda}{\Omega}\left(\left(\frac{\partial \sigma}{\partial r^{\prime}}\right)^{2}+\frac{1}{r^{\prime 2}}\left(\frac{\partial \sigma}{\partial \theta^{\prime}}\right)^{2}\right)+\Gamma\left(\frac{\partial \sigma}{\partial r^{\prime}} \frac{\partial \bar{\theta}}{\partial r^{\prime}}+\frac{1}{r^{\prime 2}} \frac{\partial \sigma}{\partial \theta^{\prime}} \frac{\partial \bar{\theta}}{\partial \theta^{\prime}}\right) \\
& +\frac{G_{c}}{\Omega}\left(\frac{\partial w^{\prime}}{\partial r^{\prime}} \frac{\partial w^{\prime}}{\partial z^{\prime}}+M \frac{\partial w^{\prime}}{\partial r^{\prime}} \frac{\partial w^{\prime}}{\partial z^{\prime}}-K\left(\frac{\partial w^{\prime}}{\partial r^{\prime}}\right)^{3} \frac{\partial w^{\prime}}{\partial z^{\prime}}\right) .
\end{aligned}
$$

By ignoring the prime symbols of the abovementioned equation, we obtain

$$
\begin{aligned}
N_{s}= & \left(\left(\frac{\partial \bar{\theta}}{\partial r}\right)^{2}+\frac{1}{r^{2}}\left(\frac{\partial \bar{\theta}}{\partial \theta}\right)^{2}\right)+\frac{\Gamma \Lambda}{\Omega}\left(\left(\frac{\partial \sigma}{\partial r}\right)^{2}+\frac{1}{r^{2}}\left(\frac{\partial \sigma}{\partial \theta}\right)^{2}\right)+\Gamma\left(\frac{\partial \sigma}{\partial r} \frac{\partial \bar{\theta}}{\partial r}+\frac{1}{r^{2}} \frac{\partial \sigma}{\partial \theta} \frac{\partial \bar{\theta}}{\partial \theta}\right) \\
& +\frac{G_{c}}{\Omega}\left(\frac{\partial w}{\partial r} \frac{\partial w}{\partial z}+M \frac{\partial w}{\partial r} \frac{\partial w}{\partial z}-K\left(\frac{\partial w}{\partial r}\right)^{3} \frac{\partial w}{\partial z}\right) .
\end{aligned}
$$

Moreover, the Bejan number $\left(B_{e}\right)$ is defined as [30]

$$
\begin{aligned}
B_{e}= & \left(\left(\frac{\partial \bar{\theta}}{\partial r}\right)^{2}+\frac{1}{r^{2}}\left(\frac{\partial \bar{\theta}}{\partial \theta}\right)^{2}\right)\left(\left(\left(\frac{\partial \bar{\theta}}{\partial r}\right)^{2}+\frac{1}{r^{2}}\left(\frac{\partial \bar{\theta}}{\partial \theta}\right)^{2}\right)+\frac{\Gamma \Lambda}{\Omega}\left(\left(\frac{\partial \sigma}{\partial r}\right)^{2}+\frac{1}{r^{2}}\left(\frac{\partial \sigma}{\partial \theta}\right)^{2}\right)\right. \\
& \left.+\Gamma\left(\frac{\partial \sigma}{\partial r} \frac{\partial \bar{\theta}}{\partial r}+\frac{1}{r^{2}} \frac{\partial \sigma}{\partial \theta} \frac{\partial \bar{\theta}}{\partial \theta}\right)+\frac{G_{c}}{\Omega}\left(\frac{\partial w}{\partial r} \frac{\partial w}{\partial z}+M \frac{\partial w}{\partial r} \frac{\partial w}{\partial z}-K\left(\frac{\partial w}{\partial r}\right)^{3} \frac{\partial w}{\partial z}\right)\right)^{-1} .
\end{aligned}
$$

\section{Discussion on Results}

This segment is assigned to giving the graphical appearance dedicating the key features of the study by drawing figures against the most pertinent parameters by taking the solution series up to the first two orders of the summation. These results also reflect the physical logic through a mathematical model of the real phenomenon. It also helps the engineers and scientist to work on such problems experimentally. The numerical data of velocity, temperature, entropy, and Bejan number profiles have been presented in Tables 1-4, respectively. From Tables 1 and 2, one can clarify that this study reproduces the results of Nadeem et al. [45] which notify the validity of the current analysis. These tables have been prepared also to estimate the variations of physical properties and imagine a difference of the behavior of Newtonian and non-Newtonian fluids under similar circumstances.

From here, we can clearly find out the viscous effects on the different viscosity model which are also manipulated latterly through graphics. The graphs for velocity of the nanofluid, temperature profile, entropy generation, and Bejan number have also been invoked in this study. The data for obtained quantities have been collected from the achieved analytical solutions. The main focus is kept on analyzing the difference of behavior shown by Newtonian $M=K=0$ and the Eyring-Powell fluid model $M=K=0.2$ under the variation of different flow factors. The velocity radial component $w$ can be understood diagrammatically from Figures 1-3. Figures 4-6 exhibit the deportment of temperature distribution $\theta$. The entropy generation analysis can be perceived from graphs of $N_{S}$ which are placed in Figures 7-10, and the Bejan number variation has been offered through Figures 11-14.

5.1. Velocity Profile. It is observed from Figure 1 that the Eyring-Powell fluid travels faster as compared to the Newtonian fluid under the variation of the Grashof number $G_{r}$. It is deeply observed that the rise in values of Grashof number $G_{r}$ shows that the peak value tends to shift toward the upper cylinder. It is for the verity that uplift in the 
TABLE 1: Comparative data of velocity $w$.

\begin{tabular}{lccc}
\hline$r$ & Nadeem et al. [45] & Current study when $K=M=0$ & Eyring-Powell fluid when $K=M=0.2$ \\
\hline 0.1 & 0.93621 & 0.93621 & 1.07305 \\
0.2 & 1.20280 & 1.20280 & 1.06557 \\
0.3 & 1.33809 & 1.33809 & 1.23787 \\
0.4 & 1.36955 & 1.36955 & 1.32323 \\
0.5 & 1.31354 & 1.31354 & 1.30876 \\
0.6 & 1.17896 & 1.17896 & 1.20033 \\
0.7 & 0.97092 & 0.97092 & 1.00403 \\
0.8 & 0.69251 & 0.69251 & 0.72443 \\
0.9 & 0.34569 & 0.34569 & 0.36479 \\
1.0 & -0.06832 & -0.06832 & -0.07258 \\
1.1 & -0.54873 & -0.54873 & -0.58602 \\
\hline
\end{tabular}

TABle 2: Comparative data of temperature profile $\theta$.

\begin{tabular}{lccc}
\hline$r$ & Nadeem et al. [45] & Current study when $K=M=0$ & Eyring-Powell fluid when $K=M=0.2$ \\
\hline 0.1 & -2.65812 & -2.65812 & 0.29081 \\
0.2 & 0.00717 & 0.00717 & 0.00323 \\
0.3 & 0.94041 & 0.94041 & 0.59033 \\
0.4 & 1.33187 & 1.33187 & 0.95761 \\
0.5 & 1.48432 & 1.48432 & 1.15508 \\
0.6 & 1.51257 & 1.51257 & 1.24405 \\
0.7 & 1.46984 & 1.46984 & 1.26306 \\
0.8 & 1.38411 & 1.38411 & 1.23571 \\
0.9 & 1.27134 & 1.27134 & 1.17683 \\
1.0 & 1.14125 & 1.14125 & 1.09610 \\
1.1 & 1.00000 & 1.00000 & 1.00000 \\
\hline
\end{tabular}

TABle 3: Comparative data of entropy generation profile $N_{s}$.

\begin{tabular}{lcc}
\hline$r$ & Newtonian fluid when $K=M=0$ & Eyring-Powell fluid when $K=M=0.2$ \\
\hline 0.1 & 50.1938 & 62.9615 \\
0.2 & 10.5518 & 12.1126 \\
0.3 & 4.34614 & 4.79125 \\
0.4 & 2.33529 & 2.51282 \\
0.5 & 1.44690 & 1.53105 \\
0.6 & 0.97960 & 1.02353 \\
0.7 & 0.70457 & 0.72868 \\
0.8 & 0.52948 & 0.54288 \\
0.9 & 0.41134 & 0.41857 \\
1.0 & 0.32800 & 0.33149 \\
1.1 & 0.26708 & 0.26822 \\
\hline
\end{tabular}

TABle 4: Comparative data of the Bejan number profile $B_{e}$.

\begin{tabular}{lcc}
\hline$r$ & Newtonian fluid when $K=M=0$ & Eyring-Powell fluid when $K=M=0.2$ \\
\hline 0.1 & 0.18389 & 0.15935 \\
0.2 & 0.33130 & 0.29225 \\
0.3 & 0.46349 & 0.41517 \\
0.4 & 0.58363 & 0.53109 \\
0.5 & 0.69574 & 0.64370 \\
0.6 & 0.80377 & 0.75691 \\
0.7 & 0.91142 & 0.87478 \\
0.8 & 1.02216 & 1.00175 \\
0.9 & 1.13951 & 1.14304 \\
1.0 & 1.26728 & 1.30530 \\
1.1 & 1.40994 & 1.49768 \\
\hline
\end{tabular}




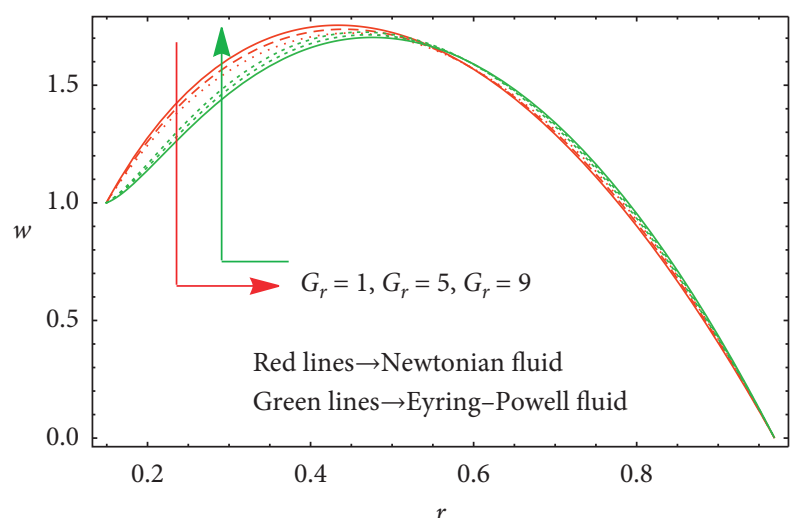

FIgURE 1: Structure of velocity factor $w$ with $G_{r}$ for fixed $\theta=0.8, \phi=0.1, B_{r}=1, \delta=0.1, Q=0.1, K=2, M=5$.

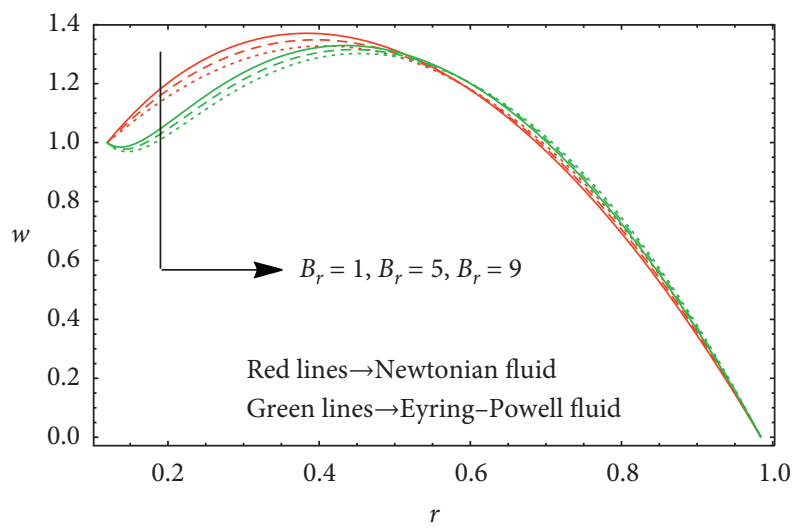

FIGURE 2: Structure of velocity factor $w$ with $B_{r}$ for fixed $\theta=0.8$, $\phi=0.1, \delta=0.1, G_{r}=1, Q=0.1, K=2, M=5$.

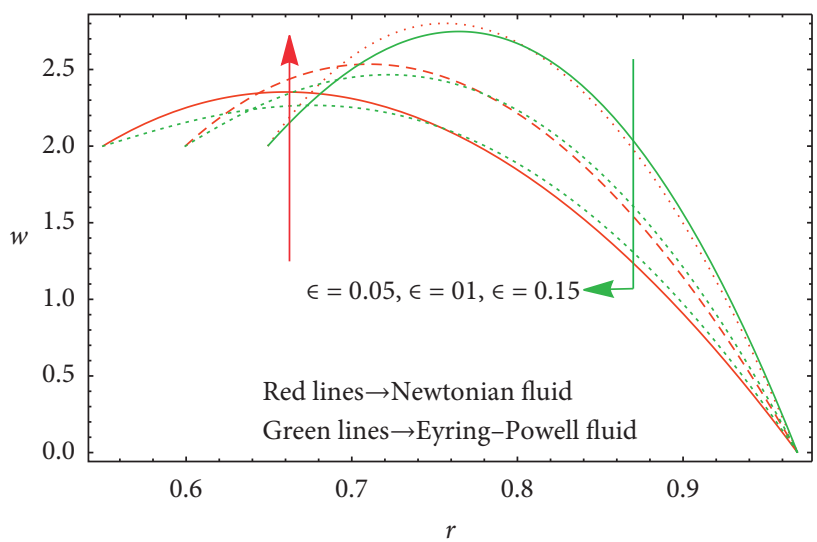

Figure 3: Structure of velocity factor $w$ with $\epsilon$ for fixed $\theta=1, \phi=$ $0.1, \delta=0.1, G_{r}=1, B_{r}=1, Q=0.25, K=0.1, M=0.1$.

values of $G_{r}$ buoyancy impacts raising the fluid, which happens to generate free convection. Normally, the density tends to rise with the reduction in temperature, but in case of the Newtonian fluid, the uniform viscosity measures the inverse behavior against the same factor. With the large

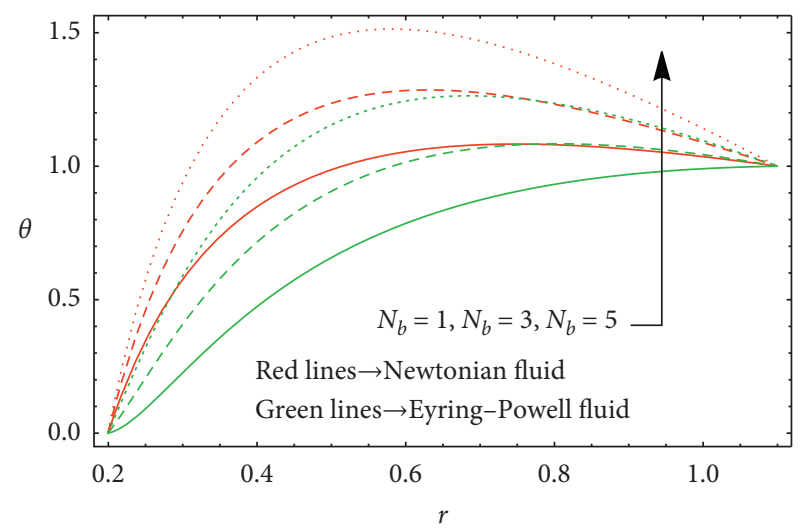

Figure 4: Alteration of heat factor $\bar{\theta}$ for $N_{b}$ when $\theta=0.1 B_{r}=$ $0.5, \delta=0.1, \phi=0.5, V=0.3, K=1, M=1, \quad G_{c}=1, N_{t}=0.2$, $\varepsilon=0.1$.

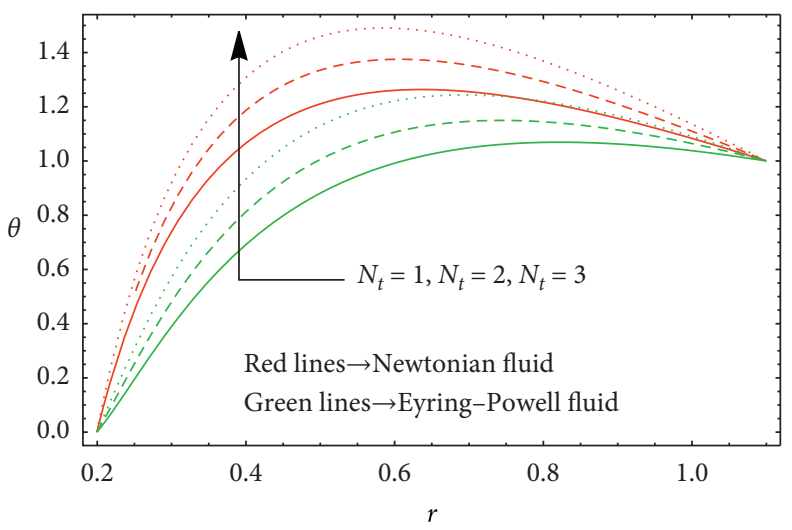

Figure 5: Alteration of heat factor $\bar{\theta}$ for $N_{t}$ when $\theta=0.1, \phi=0.5$, $N_{b}=0.2, \quad \delta=0.1, B_{r}=0.5, V=0.3, K=1, M=1, G_{c}=1, \varepsilon=$ 0.1 .

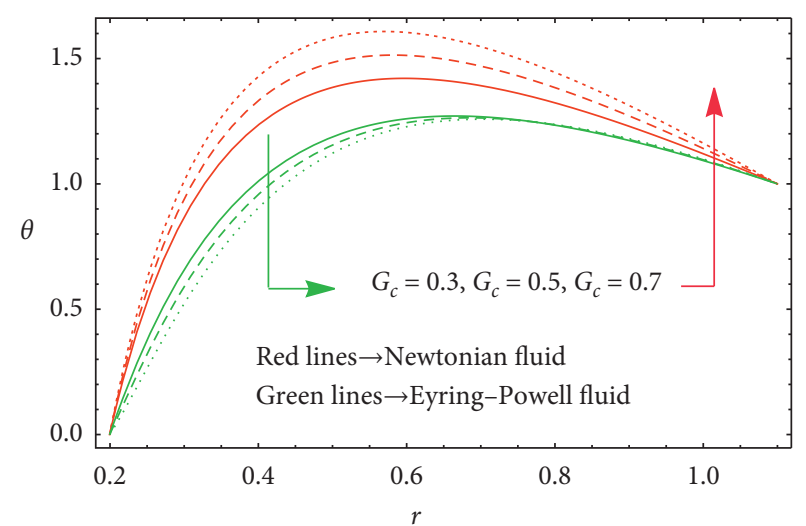

FIgURE 6: Alteration of heat factor $\bar{\theta}$ for $G_{c}$ when $\theta=0.1, \phi=0.5$, $N_{t}=0.2, \quad \delta=0.1, B_{r}=0.5, V=0.3, K=1, M=1, N_{b}=1, \varepsilon=$ 0.1 .

value of $B_{r}$, the energy dissipates due to thermal enhancement and, hence, rises the flow near the outer cylinder which are observed in Figure 2, and the same observation is reported for viscous and nonlinear fluids. In Figure 3, variation 


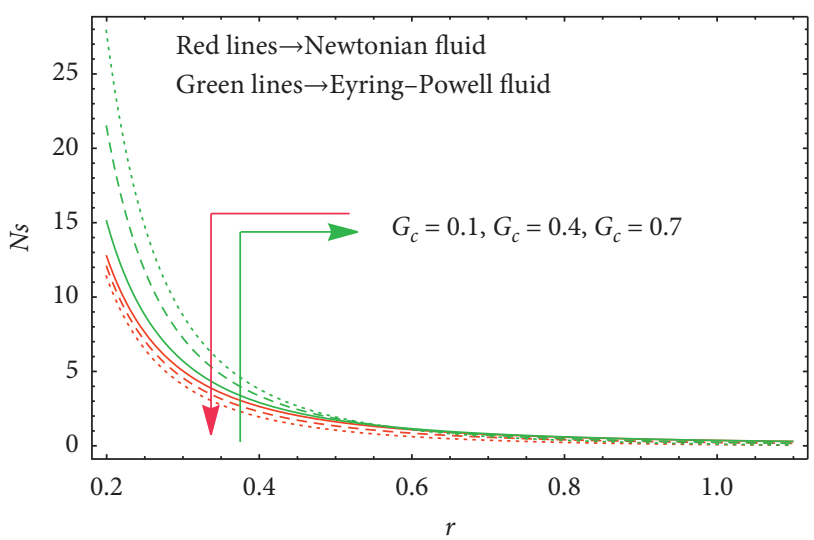

Figure 7: For $N_{s}$ with fixed $G_{c}$ where $\delta=0.01, \theta=0.1, \phi=0.8, \varepsilon=$ $0.01, \Gamma=0.4, N_{b}=0.9, N_{t}=0.5, B_{r}=1, G_{r}=1, V=0.3, K=2$, $M=1, Q=1, \Lambda=0.1, \Omega=0.3$.

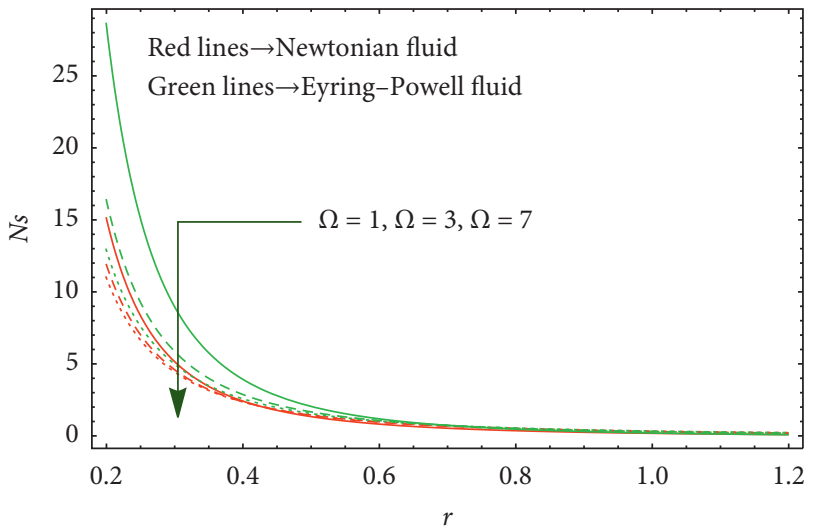

Figure 8: For $N_{s}$ with fixed $\Omega$ where $\delta=1.5, \theta=0.1, \phi=2, \varepsilon=$ $0.01, \Gamma=0.4, N_{b}=0.9, N_{t}=0.5, \quad B_{r}=1, G_{r}=5, V=0.3, K=$ $1, M=1, Q=1, \Lambda=1, G_{c}=0.01$.

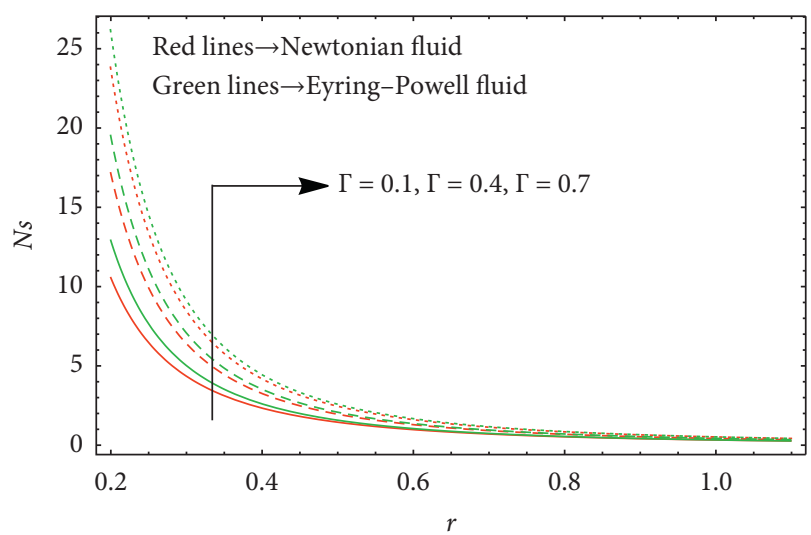

Figure 9: For $N_{s}$ with fixed $\Gamma$ where $\delta=0.01, \theta=0.3, \phi=0.8, \epsilon=$ $0.01, \mathrm{G}_{\mathrm{c}}=0.01, N_{b}=0.9, N_{t}=0.5, \quad B_{r}=1, G_{r}=3, V=0.3, K=$ $1, M=1, Q=1, \Lambda=0.4, \Omega=0.3$.

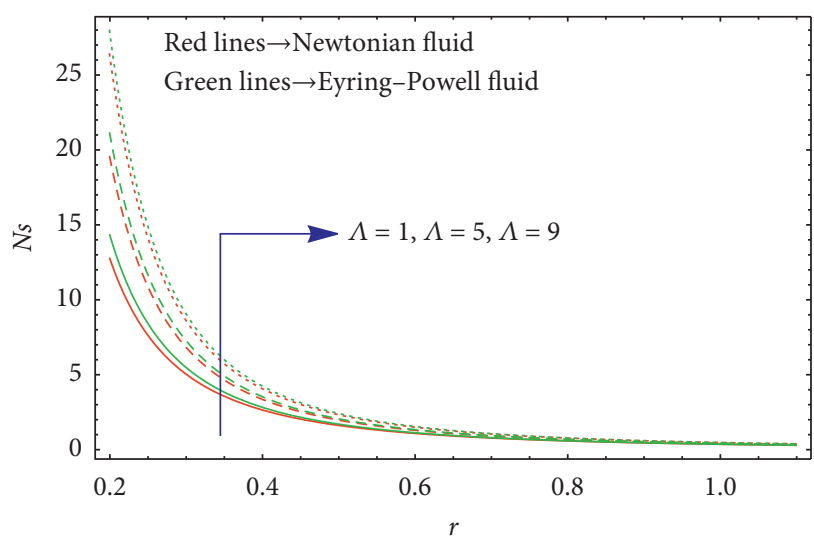

Figure 10: For $N_{s}$ with fixed $\Lambda$ where $\delta=0.01$, $\theta=0.1, \phi=0.8, \epsilon=0.1, \quad \mathrm{G}_{\mathrm{c}}=0.01, N_{b}=0.9, N_{t}=0.5, B_{r}=1$, $G_{r}=3, V=0.1, K=1, M=1, Q=1, \Gamma=0.4, \Omega=0.3$.

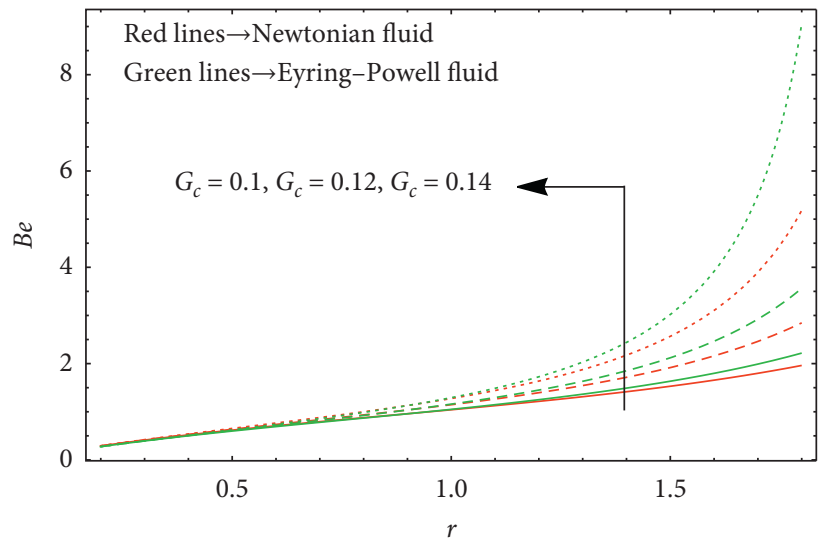

Figure 11: For $B_{e}$, Be with fixed $\mathrm{G}_{\mathrm{c}}$ where $\delta=0.1, \theta=0.1, \phi=$ $0.5, \epsilon=0.01, \Lambda=1, N_{b}=0.9, N_{t}=0.5, B_{r}=1, G_{r}=5, V=0.3$, $K=1, M=3, Q=1, \Gamma=0.4, \Omega=0.3$.

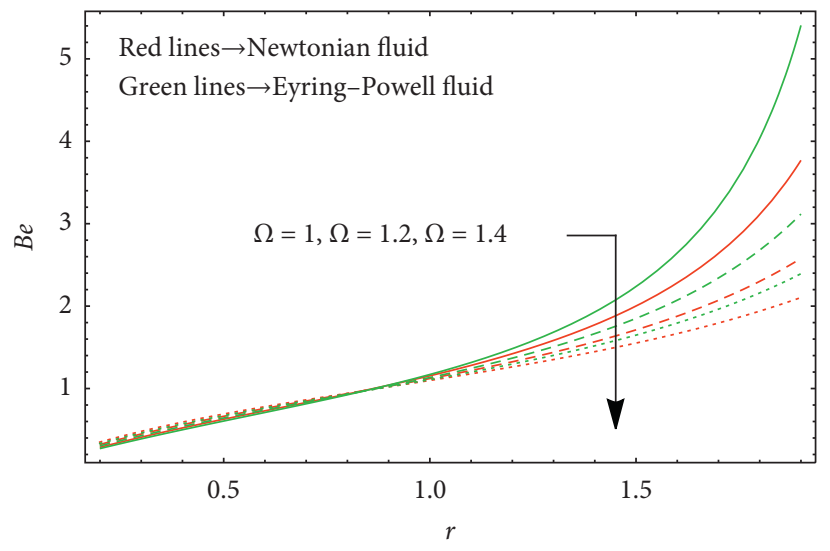

Figure 12: For $B_{e}$, Be with fixed $\Omega$ where $\delta=0.1, \theta=0.1, \phi=$ $0.5, \varepsilon=0.2, \Lambda=1, N_{b} \quad=0.9, N_{t}=0.5, B_{r}=1, G_{r}=5, V=0.3$, $K=1, M=3, Q=1, \Gamma=0.4, G_{c}=0.01$. 


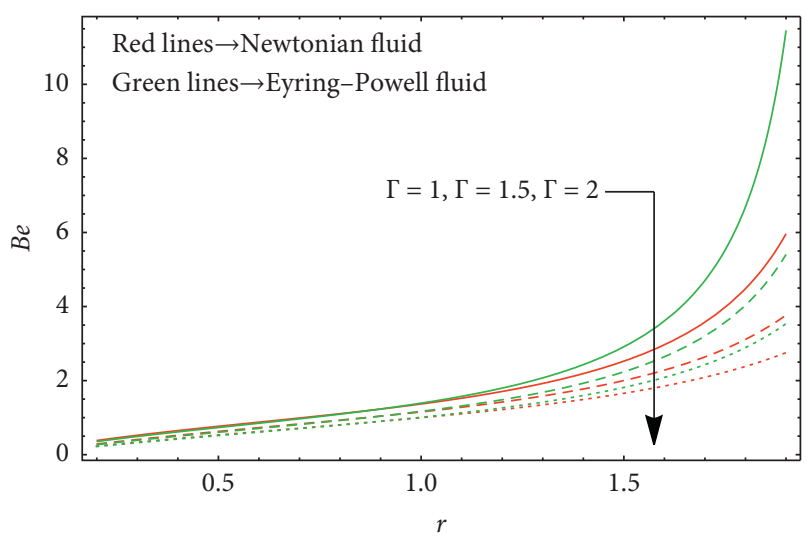

Figure 13: For $B_{e}$ with fixed $\Gamma$ where $\delta=0.1, \theta=0.1, \phi=0.5, \varepsilon=$ $0.2, \Lambda=0.1, N_{b}=0.9, N_{t}=0.5, B_{r}=5, G_{r}=1, V=0.2, K=1, M$ $=1, Q=1, \Omega=0.3, G_{c}=0.01$.

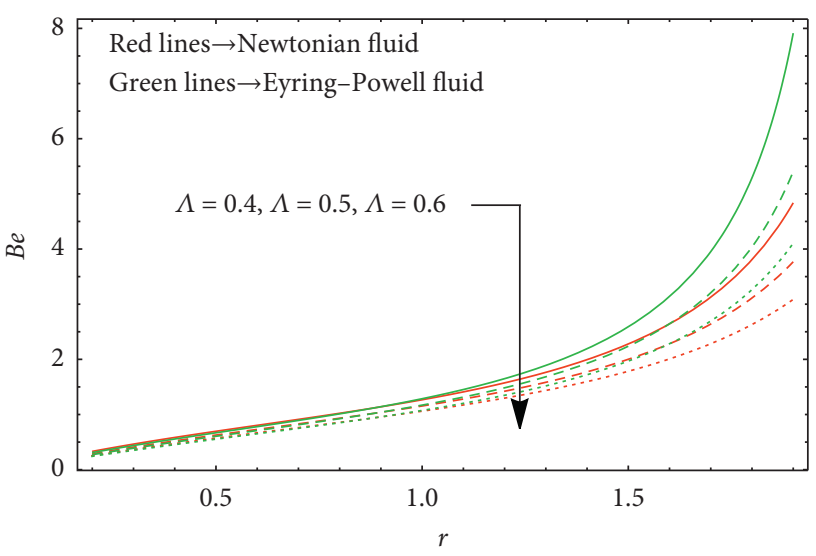

Figure 14: For $B_{e}$ with fixed $\Lambda$ where $\delta=0.1, \theta=0.1, \phi=0.5, \varepsilon=$ $0.2, \Gamma=0.4, N_{b}=0.9, N_{t}=0.5, B_{r}=1, G_{r}=5, V=0.3, K=1, M$ $=1, Q=1, \Omega=0.3, G_{c}=0.01$.

of velocity is observed for the change in eccentricity of the cylinders. It is observed that velocity declines when the eccentricity enhanced, whereas it shows the opposite behavior for the Newtonian fluid case. It is found due to the fact that Eyring-Powell fluid is higher in its viscosity with the detraction of the inner cylinder from the central axis which was not in the case of a linear model.

5.2. Temperature Dispersion Profile. The variation in temperature distribution is observed from Figures 4-6. The parameters of interest are the Brownian motion factor $N_{b}$, thermophoresis coefficient $N_{t}$, and nanoparticles Grashof factor $G_{c}$. Examining the results, we observe that a more parabolic nature is exhibited in case of temperature profile than velocity curves. With the rise in the value of the Brownian motion parameter $N_{b}$, Brownian motion increases that leads to a rise in temperature of the fluid; it is validated from Figure 4. In Figure 5, the variation of the thermophoresis parameter $N_{t}$ is observed. It is clear from here that temperature increases in the flow domain since the rise in thermal diffusion. The Brinkman number $G_{c}$ shows a reduction in temperature in the flow domain due to the rise in energy dissipation due to viscosity diffusion which is contradictory to the Newtonian fluid case where an opposite behavior is provoked (see Figure 6). In the abovementioned three discussed results, it is very obvious that temperature variation for the viscous and nonlinear model of fluid shows a similar situation against $N_{b}$ and $N_{t}$, but there are inverse calculations for the two different phases of fluid just because of the viscosity alteration aspect.

5.3. Entropy Measurements. The factor which is the most important one for thermal analysis of liquid flow is the dissipation analysis of energy which is defined by the entropy generation parameter $\left(N_{s}\right)$. The variation in entropy generation is graphically displayed for various values $G_{C}, \Omega, \Gamma$, and $\Lambda$ in Figures 7-10, respectively. Entropy generation decreases for larger values of the Brinkman number $G_{C}$ since temperature profile shows a rise for $G_{c}$ (Figure 7). From Figure 8, the findings made are described as with the rise in the concentration parameter, entropy decreases. An opposite behavior of the temperature parameter $\Gamma$ is observed from Figure 9. From Figure 10, it is reflected that the ratio of the concentration parameter $\Lambda$ is responsible for higher entropy generation. From Figures 7-10, the interesting aspect which is pointed out is that only $G_{c}$ puts opposite impacts on viscous fluid but other parameters make no prominent change for the Newtonian fluid, and an important notable thing is that entropy peaks are measured in the neighborhood of the inner cylinder which becomes flat at the surface of the outer one.

5.4. Bejan Number Curves. A graphical representation of $B_{e}$ is displayed in Figures 11-14. It is defined as the entropy generation due to heat loss divided by total entropy. It is observed from Figure 11 that, with a rise in the Brinkman number $G_{c}$, the Bejan Number $B_{e}$ increases due to the increase in thermal entropy for the rise in temperature which comes from high values of the Brinkman number. It indicates that the contribution of $G_{c}$ in entropy increases with $r$ for higher values of $G_{c}$. Figure 12 shows a decrease in the entropy ratio for large values of $\Omega$, which means that an increase in the concentration parameter produces less entropy due to thermal transfer and more entropy due to collective aspects generating entropy, whereas the temperature parameter $\Gamma$ and temperature to concentration parameter $\Lambda$ show an opposite behavior than the Brinkman number as seen from Figures 13 and 14, respectively. On the parallel side, these plots also investigate that the Bejan number curves are reflecting similar results for both the viscous and nonlinear fluids. The only thing that is prominent from these graphs is that the thermal entropy is maximum near the outer cylinder.

\section{Concluding Remarks}

In the current paper, we have elaborated entropy measures occurring during the biological movement of a viscoelastictype fluid in between an annular region of two cylinders 
having distinct centers. The nanoparticles phenomenon is also taken into consideration. The equations have been assembled in the light of the lubrication strategy and steady format by transformation used in a newly arranged wave frame of reference. In terms of solution, expressions have been analytically gathered with the help of HPM and numerical integration is operated to the expression of pressure rise on mathematical computing the Mathematica tool. The expressions of the entropy generation factor and Bejan number are incorporated by obtained data of viscous dissipation, heat, and energy terms. Comparison of two different models of the fluid is kept under key observation in all figures. The final headings extracted from the whole analysis are reflected in the following:

(i) It is found that the velocity of the substance is getting lower by increasing eccentricity measures of the cylinders and the local temperature Grashof number but higher in front of the local nanoparticles Grashof number. It is also seen that the Newtonian fluid shows opposite readings against the eccentricity and local nanoparticles Grashof number but the same for the temperature Grashof number

(ii) Temperature of the fluid is enhanced for both of the factors, the Brownian motion parameter and thermophoresis parameter, but suppressed against the Brinkman number which is a contradictory result in the Newtonian fluid

(iii) It is noted that entropy generation gets lowered with the increase in the concentration parameter, but it is enhanced under the variation of the Brinkman number, temperature variance representative, and the factor showing the ratio of the thermal factor and concentration factors, but the difference of variation for the viscous fluid is only reported in the case of the Brinkman number

(iv) Bejan number curves are showing totally inverse characteristics than the entropy generation curves

(v) It can be resulted from abovementioned detailed discussion that the Eyring-Powell fluid shows the difference of behavior mostly for viscous dissipation effects in entropy generation and in the presence of nanoparticles, and it travels faster than the viscous fluid

(vi) This study is validated by the result of Nadeem et al. [45] and also compares the characteristics of both the viscous and Eyring-Powell fluid model through special cases $K=M=0$

\section{Abbreviations}

$S_{\mathrm{LM}}: \quad$ Stress tensor of the Eyring-Powell fluid

$\mu$ : $\quad$ Viscosity of the fluid

$V_{p}: \quad$ Velocity field

$x^{i}$ : $\quad$ Coordinates in fixed frame

$\beta, c: \quad$ Material constants for the fluid

p: $\quad$ Pressure term

$\begin{array}{ll}a: & \text { Radius of the outer tube } \\ c_{1}: & \text { Wave speed } \\ \lambda: & \text { Wavelength } \\ \delta: & \text { Radius of the inner tube } \\ t: & \text { Time factor } \\ u, v, w: & \text { Dimensionless velocity components } \\ r, \theta, z: & \text { Dimensionless cylindrical coordinates } \\ b: & \text { Wave amplitude } \\ \epsilon: & \text { Dimensionless eccentricity factor } \\ R_{e}: & \text { Reynolds number } \\ T, T_{0}, T_{1}: & \text { Temperature factors } \\ C, C_{0}, C_{1}: \text { Nanoparticles concentration } \\ (\rho c)_{f}: & \text { Fluid heat capacity } \\ (\rho c)_{p}: & \text { Nanoparticles heat capacity } \\ P_{r}: & \text { Prandtl number } \\ S_{c}: & \text { Schmidt number } \\ k: & \text { Thermal conductivity of the fluid } \\ B_{r}: & \text { Local temperature Grashof number } \\ G_{r}: & \text { Local nanoparticle Grashof number } \\ N_{b}: & \text { Brownian motion parameter } \\ N_{t}: & \text { Thermophoresis parameter } \\ G_{c}: & \text { Brinkman number } \\ M, K: & \text { Eyring-Powell dimensionless factors } \\ V: & \text { Velocity of the inner tube } \\ r_{1}, r_{2}: & \text { Inner and outer boundary functions } \\ q^{\prime \prime}: & \text { Homotopy parameter } \\ \mathscr{L}: & \text { Linear operator } \\ \bar{\theta}: & \text { Dimensionless temperature } \\ \sigma: & \text { Dimensionless volume fraction. }\end{array}$

\section{Data Availability}

No data were used to support this study.

\section{Conflicts of Interest}

The authors declare no conflicts of interest.

\section{Authors' Contributions}

All the authors contributed equally.

\section{Acknowledgments}

The authors are thankful to the University of Education, Lahore, Pakistan, for the support.

\section{References}

[1] M. B. Arain, M. M. Bhatti, A. Zeeshan, T. Saeed, and A. Hobiny, "Analysis of arrhenius kinetics on multiphase flow between a pair of rotating circular plates," Mathematical Problems in Engineering, vol. 2020, Article ID 2749105, 17 pages, 2020.

[2] M. Irfan, M. A. Farooq, A. Mushtaq, and Z. H. Shamsi, "Unsteady MHD bionanofluid flow in a porous medium with thermal radiation near a stretching/shrinking sheet," Mathematical Problems in Engineering, vol. 2020, Article ID 8822999, 14 pages, 2020. 
[3] H. Maleki, M. R. Safaei, A. A. A. A. Alrashed, and A. Kasaeian, "Flow and heat transfer in non-Newtonian nanofluids over porous surfaces," Journal of Thermal Analysis and Calorimetry, vol. 135, no. 3, pp. 1655-1666, 2019.

[4] H. Maleki, M. R. Safaei, H. Togun, and M. Dahari, "Heat transfer and fluid flow of pseudo-plastic nanofluid over a moving permeable plate with viscous dissipation and heat absorption/generation," Journal of Thermal Analysis and Calorimetry, vol. 135, no. 3, pp. 1643-1654, 2019.

[5] H. K. Yoon, An experimental and analytical study of heat transfer to polymer solutions in turbulent pipe flows under constant wall heat flux, Ph.D. thesis, Oklahoma State University, Stillwater, OK, USA, 1986.

[6] E. Y. Kwack, Effect of Weissenberg number on turbulent heat transfer and friction factor of viscoelastic fluids, Ph.D. thesis, University of Illinois at Chicago, Chicago, IL, USA, 1983.

[7] M. Khan, M. Irfan, W. A. Khan, and A. S. Alshomrani, "A new modeling for 3D Carreau fluid flow considering nonlinear thermal radiation," Results in Physics, vol. 7, pp. 2692-2704, 2017.

[8] S. Matsuhisa and R. B. Bird, "Analytical and numerical solutions for laminar flow of the non-Newtonian ellis fluid," AIChE Journal, vol. 11, no. 4, pp. 588-595, 1965.

[9] H. K. Yoon and A. J. Ghajar, "A note on the Powell-Eyring fluid model," International Communications in Heat and Mass Transfer, vol. 14, no. 4, pp. 381-390, 1987.

[10] M. Patel and M. G. Timol, "Numerical treatment of Powell-Eyring fluid flow using method of satisfaction of asymptotic boundary conditions (MSABC)," Applied Numerical Mathematics, vol. 59, no. 10, p. 2592, 2009.

[11] T. Hayat, M. Farooq, A. Alsaedi, and Z. Iqbal, "Melting heat transfer in the stagnation point flow of powell-Eyring fluid," Journal of Thermophysics and Heat Transfer, vol. 27, no. 4, pp. 761-766, 2013.

[12] M. Jalil, S. Asghar, and S. M. Imran, "Self similar solutions for the flow and heat transfer of Powell-Eyring fluid over a moving surface in a parallel free stream," International Journal of Heat and Mass Transfer, vol. 65, pp. 73-79, 2013.

[13] S. Islam, A. Shah, C. Y. Zhou, and I. Ali, "Homotopy perturbation analysis of slider bearing with Powell-Eyring fluid," Zeitschrift für Angewandte Mathematik und Physik, vol. 60, no. 6, p. 1178, 2009.

[14] S. Hina, M. Mustafa, T. Hayat, and A. Alsaedi, "Peristaltic transport of Powell-Eyring fluid in a curved channel with heat/mass transfer and wall properties," International Journal of Heat and Mass Transfer, vol. 101, pp. 156-165, 2016.

[15] T. Hayat, Z. Hussain, M. Farooq, and A. Alsaedi, "Magnetohydrodynamic flow of Powell-Eyring fluid by a stretching cylinder with Newtonian heating," Thermal Science, vol. 22, no. 1, pp. 371-382, 2018.

[16] A. H. Shapiro, "Pumping and retrograde diffusion in peristaltic waves," in Proceedings of the Workshop in Ureteral Reflux in Children, pp. 109-126, Washington, DC, USA, 1967.

[17] T. W. Latham, "Fluid motions in a peristaltic pump," Doctoral dissertation, Massa-Chusetts Institute of Technology, Cambridge, MA, USA, 1966.

[18] M. Y. Jaffrin and A. H. Shapiro, "Peristaltic pumping," Annual Review of Fluid Mechanics, vol. 3, no. 1, pp. 13-37, 1961.

[19] L. Zhang, M. M. Bhatti, M. Marin, and K. S. Mekheimer, "Entropy analysis on the blood flow through anisotropically tapered arteries filled with magnetic zinc-oxide $(\mathrm{ZnO})$ nanoparticles," Entropy, vol. 22, no. 10, p. 1070, 2020.

[20] K. Javid, M. Hassan, M. Imran Asjad et al., "Rheological effects of biomimetic propulsion on fluid flow: an application of bio- engineering," European Physical Journal Plus, vol. 134, p. 522, 2019.

[21] R. Ellahi, M. M. Bhatti, and C. M. Khalique, "Three-dimensional flow analysis of Carreau fluid model induced by peristaltic wave in the presence of magnetic field," Journal of Molecular Liquids, vol. 241, pp. 1059-1068, 2017.

[22] T. Hayat, B. Ahmed, A. Alsaedi, and F. M. Abbasi, "Numerical study for peristalsis of Carreau-Yasuda nanomaterial with convective and zero mass flux condition," Results in Physics, vol. 8, pp. 1168-1177, 2018.

[23] J. Prakash and D. Tripathi, "Electroosmotic flow of Williamson ionic nanoliquids in a tapered microfluidic channel in presence of thermal radiation and peristalsis," Journal of Molecular Liquids, vol. 256, pp. 352-371, 2018.

[24] A. Zeeshan, N. Ijaz, T. Abbas, and R. Ellahi, "The sustainable characteristic of bio-bi-phase flow of peristaltic transport of MHD Jeffrey fluid in the human body," Sustainability, vol. 10, no. 8, p. 2671, 2018.

[25] A. Tanveer, T. Hayat, F. Alsaadi, and A. Alsaedi, "Mixed convection peristaltic flow of Eyring-Powell nanofluid in a curved channel with compliant walls," Computers in Biology and Medicine, vol. 82, pp. 71-79, 2017.

[26] M. M. Bhatti, A. Zeeshan, R. Ellahi, and G. C. Shit, "Mathematical modeling of heat and mass transfer effects on MHD peristaltic propulsion of two-phase flow through a DarcyBrinkman-Forchheimer porous medium," Advanced Powder Technology, vol. 29, no. 5, pp. 1189-1197, 2018.

[27] M. Afridi, M. Qasim, A. Wakif, and A. Hussanan, "Second law analysis of dissipative nanofluid flow over a curved surface in the presence of Lorentz force: utilization of the ChebyshevGauss-Lobatto spectral method," Nanomaterials, vol. 9, no. 2, p. 195, 2019.

[28] S. Nazari, R. Ellahi, M. M. Sarafraz, M. R. Safaei, A. Asgari, and O. A. Akbari, "Numerical study on mixed convection of a non-Newtonian nanofluid with porous media in a two liddriven square cavity," Journal of Thermal Analysis and Calorimetry, vol. 140, pp. 1121-1141, 2019.

[29] S. R. R. Reddy, P. B. A. Reddy, and A. M. Rashad, "Activation energy impact on chemically reacting Eyring-Powell nanofluid flow over a stretching cylinder," Arabian Journal for Science and Engineering, vol. 45, pp. 5227-5242, 2020.

[30] A. Riaz, A. Gul, I. Khan et al., "Mathematical analysis of entropy generation in the flow of viscoelastic nanofluid through an annular region of two asymmetric annuli having flexible surfaces," Coatings, vol. 10, no. 3, p. 213, 2020.

[31] M. Sheikholeslami, M. Jafaryar, S. Saleem, Z. Li, A. Shafee, and Y. Jiang, "Nanofluid heat transfer augmentation and exergy loss inside a pipe equipped with innovative turbulators," International Journal of Heat and Mass Transfer, vol. 126, pp. 156-163, 2018.

[32] S. U. Choi, "Nanofluids: from vision to reality through research," Journal of Heat Transfer, vol. 131, no. 3, 2009.

[33] R. K. Shukla and V. K. Dhir, "Numerical study of the effective thermal conductivity of nanofluids," in Proceedings of the ASME 2005 Summer Heat Transfer Conference Collocated with the ASME 2005 Pacific Rim Technical Conference and Exhibition on Integration and Packaging of MEMS, NEMS, and Electronic Systems, pp. 449-457, American Society of Mechanical Engineers Digital Collection, San Francisco, CA, USA, July 2005.

[34] A. Behzadmehr, M. Saffar-Avval, and N. Galanis, "Prediction of turbulent forced convection of a nanofluid in a tube with uniform heat flux using a two phase approach," International 
Journal of Heat and Fluid Flow, vol. 28, no. 2, pp. 211-219, 2007.

[35] J. Buongiorno, "Convective transport in nanofluids," Journal of Heat Transfer, vol. 128, no. 3, pp. 240-250, 2006.

[36] Y. M. Chu, U. Khan, A. Zaib, and S. H. Shah, "Numerical and computer simulations of cross-flow in the streamwise direction through a moving surface comprising the significant impacts of viscous dissipation and magnetic fields: stability analysis and dual solutions," Mathematical Problems in Engineering, vol. 2020, Article ID 8542396, 11 pages, 2020.

[37] A. Riaz, H. Alolaiyan, and A. Razaq, "Convective heat transfer and magnetohydro-dynamics across a peristaltic channel coated with nonlinear nanofluid," Coatings, vol. 9, no. 2, p. 816, 2019.

[38] U. Rashid, T. Abdeljawad, H. Liang, A. Iqbal, M. Abbas, and M. Siddiqui, "The shape effect of gold nanoparticles on squeezing nanofluid flow and heat transfer between parallel plates," Mathematical Problems in Engineering, vol. 2020, Article ID 9584854, 12 pages, 2020.

[39] S. U. Khan, K. Al-Khaled, and M. M. Bhatti, "Bioconvection analysis for flow of Oldroyd-B nanofluid configured by a convectively heated surface with partial slip effects," Surfaces and Interfaces, vol. 23, Article ID 100982, 2021.

[40] R. Naz, M. Noor, Z. Shah, M. Sohail, P. Kumam, and P. Thounthong, "Entropy generation optimization in MHD pseudoplastic fluid comprising motile microorganisms with stratification effect," Alexandria Engineering Journal, vol. 59, no. 1, pp. 485-496, 2020.

[41] M. A. Abbas and I. Hussain, "Statistical analysis of the mathematical model of entropy generation of magnetized nanofluid," Inventions, vol. 4, no. 2, p. 32, 2019.

[42] M. Abbas, Y. Bai, M. Rashidi, and M. Bhatti, "Analysis of entropy generation in the flow of peristaltic nanofluids in channels with compliant walls," Entropy, vol. 18, no. 3, p. 90, 2016.

[43] R. Ellahi, M. Raza, and N. S. Akbar, "Study of peristaltic flow of nanofluid with entropy generation in a porous medium," Journal of Porous Media, vol. 20, no. 5, 2017.

[44] T. Hayat, S. Nawaz, and A. Alsaedi, "Entropy generation and endoscopic effects on peristalsis with modified Darcy's law," Physica A: Statistical Mechanics and Its Applications, vol. 536, p. 120846, 2019.

[45] S. Nadeem, A. Riaz, R. Ellahi, and N. S. Akbar, "Effects of heat and mass transfer on peristaltic flow of a nanofluid between eccentric cylinders," Applied Nanoscience, vol. 4, no. 4, pp. 393-404, 2014. 